\title{
SAR-BASED WIND FIELDS OVER OFFSHORE WIND FARMS - A VALUABLE TOOL FOR PLANNING, MONITORING AND OPTIMIZATION
}

\author{
Sven Jacobsen, Andrey Pleskachevsky, Suman Singha, Anja Frost, Domenico Velotto \\ German Aerospace Center (Deutsches Zentrum für Luft- und Raumfahrt; DLR) \\ Maritime Safety and Security Lab Bremen, Germany
}

\begin{abstract}
The number of offshore wind facilities is increasing with a proportionate decline in fossil and nuclear power production. The study of turbulent wakes inside a turbine cluster is a very important topic in order to optimize cluster layout for power production. With an increasing density of wind farms in the exclusive economic zone (EEZ) of a country, shadowing effects of wind farms on adjacent clusters are becoming an important issue for wind farm performance and need to be investigated to improve power harvest predictions. We present a comparative study of wind fields of different resolutions and coverages derived from TerraSAR-X and Sentinel-1 images. We elucidate the benefits of certain data for particular applications.
\end{abstract}

Index Terms — Energy, Wind, SAR, Renewable, Power

\section{INTRODUCTION}

The increasing popularity of renewable energy resources has promoted the construction of offshore wind farms e.g. in the North Sea. While the wind farm layout consists of an array of large turbines, the interrelation of turbine wind wakes with the remaining array is of substantial interest to optimize energy yields. On the other hand, shadowing effects of wind farms on adjacent turbine clusters can be a concern for downwind located sites and strongly alter the power production and long-term calculations of depreciation costs. The downstream spatial evolution of turbulent wind wakes is very complex and depends on manifold parameters such as wind speed, wind direction, ambient atmospheric stability conditions and surface roughness. To complement and validate existing numerical models, corresponding observations are needed.

While in-situ measurements with e.g. anemometers provide a time-series at the given location, the merits of ground-based and space- or airborne remote sensing techniques are indisputable in terms of spatial coverage. Active microwave devices, such as scatterometer and synthetic aperture radar (SAR), have proven their capabilities of providing sea surface wind measurements and particularly SAR images reveal wind variations at a high spatial resolution while retaining the large coverage.

A recent study confirms the accuracy of SAR-based wind fields and the capability of deducing wind variations up to turbine height from sea surface roughness and the associated radar backscatter [1].

Currently, multiple imaging SAR platforms such as the Sentinel-1 satellites, the TanDEM-X configuration, CosmoSkymed, Radarsat 2 and ALOS-II and more are orbiting the earth. While the combination of the sources increases temporal data coverage of the area of interest, sensor- and data-specific characteristics do not always lead to comparable wind speeds derived from different sources. The quantification of these differences is a crucial component in the reliable monitoring of offshore wind farms and their impacts.

Sentinel-1 (S1) and TerraSAR-X (TSX) possess orbits, that regularly allow observation of the same area with both satellites less than 30 Minutes apart. As the satellites are also operating in different bands and at different resolutions, the comparison of S1 and TSX represents a good opportunity to investigate different sources of possible inconsistencies.

\section{METHODS AND DATA}

The deduction of wind conditions is based on a geophysical model function (GMF) which relates the observed radar backscatter to wind speed and direction and which is characteristic for the frequency band of the sensor. The necessary adaptions of the GMF coefficients to certain radar bands account for the differences in the characteristic sensitivity to certain small-scale sea surface roughness features induced mainly by wind, but also by rain or other phenomena. GMFs were initially developed for scatterometers with large footprints and low incidence angles. These GMFs are meanwhile also applied to analyze imaging SAR data with much higher spatial resolution and a wide range of incidence angles. Down to which resolution the GMFs yield reliable results is still an unresolved question.

A comparison at high spatial resolution of independent measurements by two sensors provides insight into 


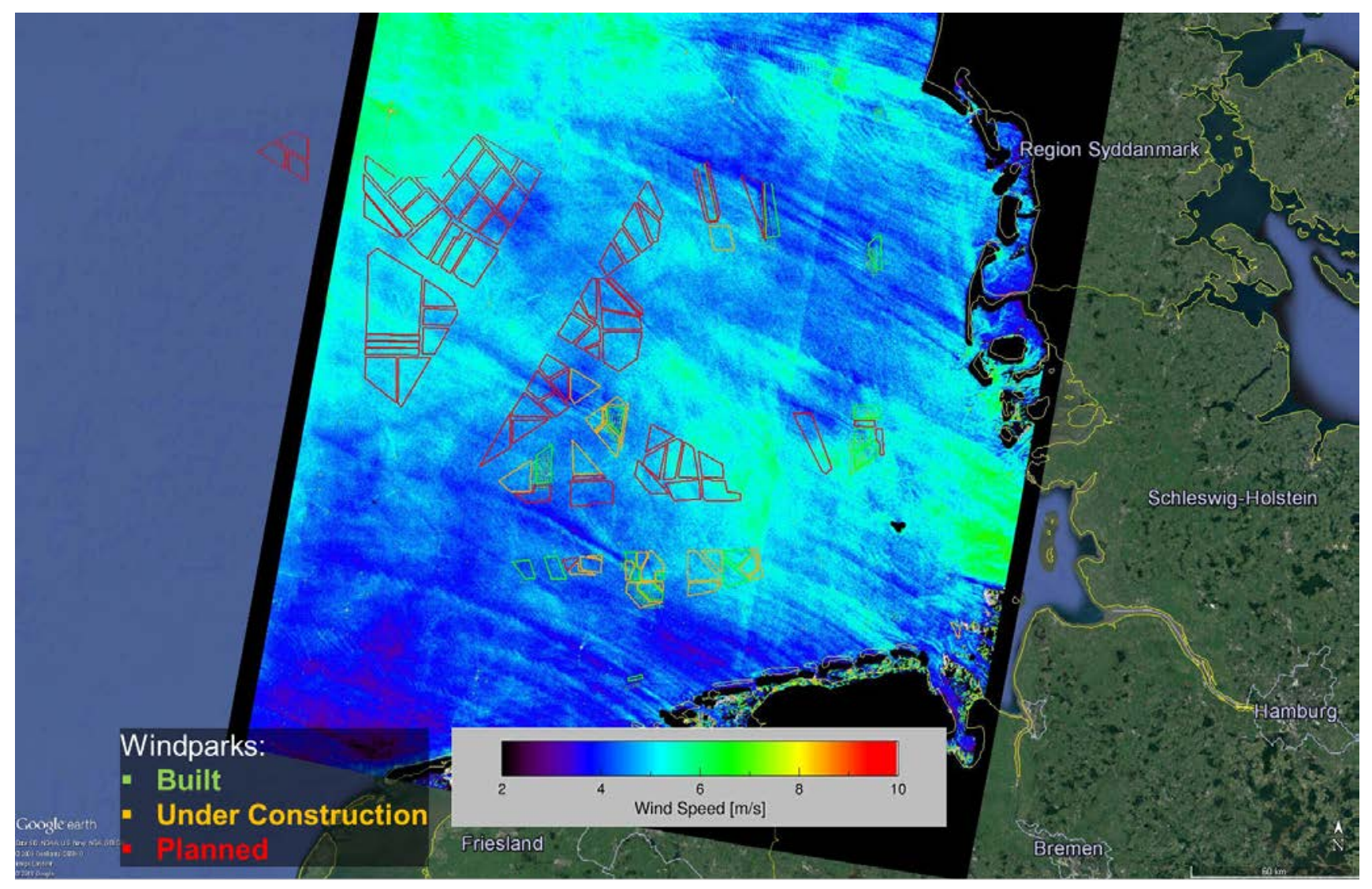

Figure 1: Sentinel-1 wind field (IW VV, June 05, 2015 ) of the German Bight and german offshore wind farm outlines as Google Earth overlay. Winds are coming from south-easterly directions and long extending wind wakes behind green outlines of operational wind farms are visible.

differences in the sensitivity to certain sea surface roughness features depending on the sensor band.

For the comparison, we use a set of X-band data acquired by TSX and C-band images from S1 that are overlapping in space and separated in time less than 30 minutes. We complement the satellite data with model results and buoy or LiDAR data where possible. We alter the wind analysis resolution and juxtapose the produced wind fields. We hereby help identify confidence margins for SAR-based wind field resolutions and find restrictions for the finest resolvable features for the given sensor.

\section{EXEMPLARY RESULTS}

In this section we exemplarily present wind fields deduced from TSX and S1 images. The wind data derived illustrates the benefits of a combination of different sensors to investigate the whole range from small-scale to large-scale wind features.

In Figure 1, a Sentinel-1 wind field in $200 \mathrm{~m}$ resolution (derived from an interferometric wide swath (IW) image in VV polarization) of the German Bight with $250 \mathrm{~km}$ swath width is shown. We follow [2] and use the CMOD5.N [3]
GMF with an offset for the wind analysis of S1 VV data. Wind farm wakes are originating from operational turbine arrays (green outlines) and extend up to $80 \mathrm{~km}$ downwind. Other wind farms that are not yet built (orange and red colored outlines) would observe a significant drop in power production as the power production is cubically related to wind speed.

In Figure 2, a TSX wind field around the Riffgat wind farm near the german island Borkum is shown. The GMF applied is XMOD2 described in [4]. The turbine wakes exhibit wind variations most likely associated with Kármán vortex streets. The measurement of the extent and spacing between the vortices is valuable for the validation and improvement of wind turbine induced turbulence models and can help optimize turbine array layouts.

\section{SUMMARY AND OUTLOOK}

A large number and high density of wind facilities stresses the importance of an investigation of inter- and intra-cluster interactions. In our work, we find that SAR-based wind fields represent a valuable data source in the monitoring of wind farms. They can contribute to constrain power 


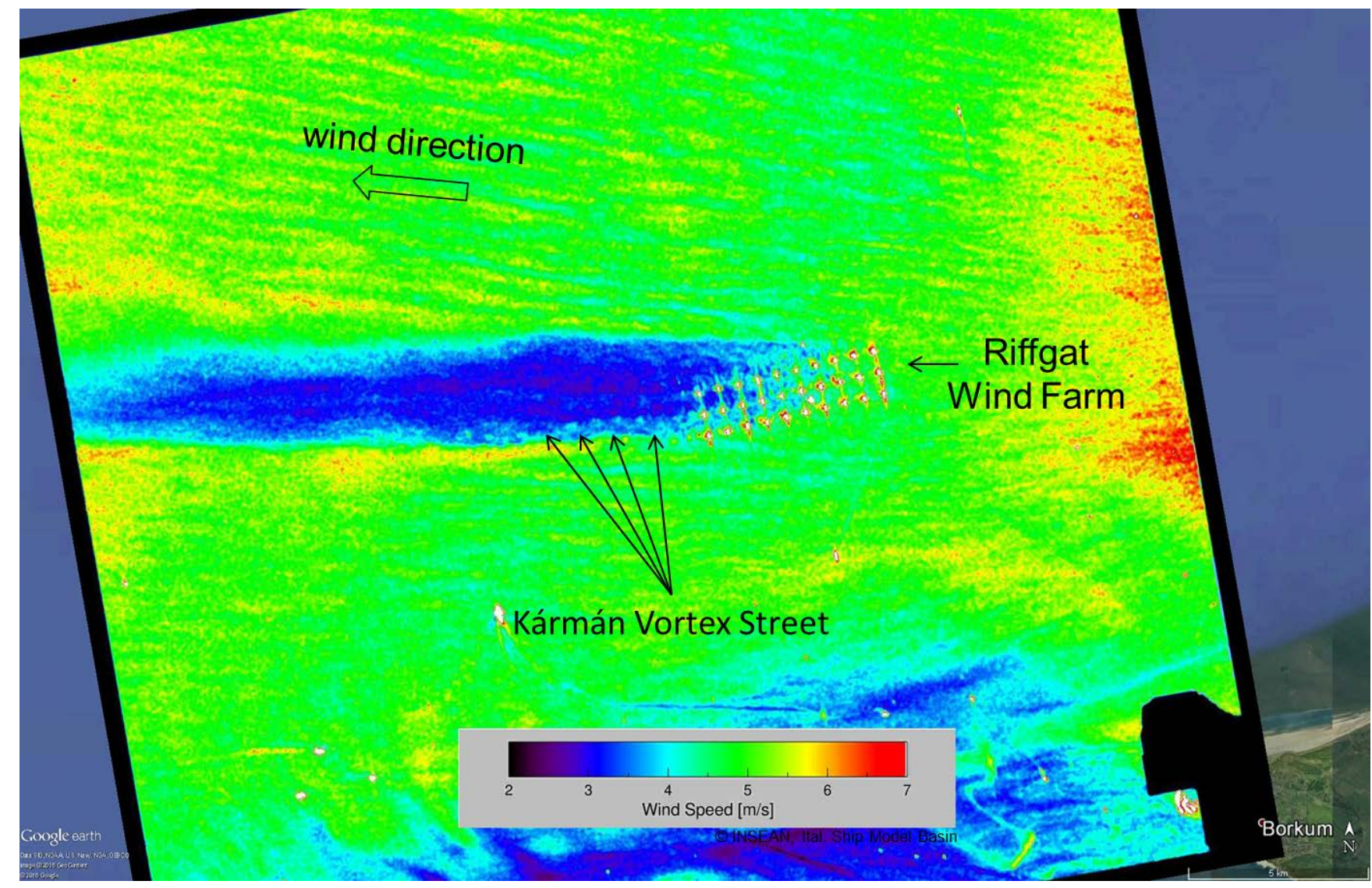

Figure 2: TSX (StripMap Image from August 20, 2015) wind field of 60m resolution over the Riffgat Wind Farm as Google Earth overlay. Within the wind shadow, turbulent structures appear, that are consistent with the characteristics of a Kármán vortex street.

production predictions and improve electricity supply reliability in a heterogeneous mix of renewable energies and fossil or nuclear fuels.

\section{REFERENCES}

[1] S. Jacobsen, S. Lehner, J. Hieronimus, J. Schneemann, and M. Kühn, "Joint Offshore Wind Field Monitoring With Spaceborne SAR and Platform-Based Doppler Lidar Measurements.," in International Archives of the Photogrammetry, Remote Sensing \& Spatial Information Sciences, 2015.

[2] F. Monaldo, C. Jackson, X. Li, and W. G. Pichel, "Preliminary Evaluation of Sentinel-1A Wind Speed Retrievals,” IEEE Journal of Selected Topics in Applied Earth Observations and Remote Sensing, vol. PP, no. 99, pp. 1-10, 2016.

[3]H. Hersbach, "Comparison of C-Band Scatterometer CMOD5.N Equivalent Neutral Winds with ECMWF,” J. Atmos. Oceanic Technol., vol. 27, no. 4, pp. 721-736, Dec. 2009.

[4] X.-M. Li and S. Lehner, "Algorithm for Sea Surface Wind Retrieval From TerraSAR-X and TanDEM-X Data," IEEE Transactions on Geoscience and Remote Sensing, vol. Early Access Online, 2013. 